\title{
A Case of Tracheomalacia in Chronic Obstructive Pulmonary Disease: What Went Wrong?
}

\author{
Carmen Pais ${ }^{1}$, Rita Carneiro Silva ${ }^{1}$, Teresa Gomes $^{2}$, Sónia Carvalho ${ }^{1}$ \\ ${ }^{1}$ Internal Medicine, Centro Hospitalar de Trás-os-Montes e Alto Douro, Unidade Hospitalar de Vila Real, Vila Real, Portugal \\ ${ }^{2}$ Pneumology, Centro Hospitalar de Trás-os-Montes e Alto Douro, Unidade Hospitalar de Vila Real, Vila Real, Portugal
}

How to cite this article: Pais C, Silva Carneiro R, Gomes T, Carlvaho S. A case of tracheomalacia in chronic obstructive pulmonary disease: what went wrong? EJCRIM 2018;5: doi:10.12890/2018_000906.

Conflicts of Interests: The Authors declare that there are no competing interests.

This article is licensed under a Commons Attribution Non-Commercial 4.0 License

\section{ABSTRACT}

Tracheobronchomalacia is defined as loss of the structural integrity of airway wall cartilaginous structures with hyperdynamic airway collapse during respiration. It is a common finding in chronic obstructive pulmonary disease (COPD) but is not always symptomatic, especially if airway narrowing is mild. Symptoms and signs develop as the severity of airway narrowing progresses. When a patient is symptomatic, a prompt study with computerized tomography and flexible bronchoscopy is mandatory for future management. We present a case of tracheobronchomalacia in a patient with COPD whose diagnosis and treatment were challenging.

\section{LEARNING POINTS}

- Tracheobroncomalacia is a common finding in chronic obstructive pulmonary disease patients; management of the obstructive disorder is the first treatment step.

- When patients remain symptomatic, non-invasive positive pressure ventilation can be applied; if there is no response, a stent trial or surgical procedure should be considered.

- It is a challenge to identify which patients should undergo invasive intervention or surgical management.

\section{KEYWORDS}

Tracheobroncomalacia, airway obstruction, bronchoscopy

\section{CASE DESCRIPTION}

We present the case of a 76-year-old man with a history of chronic obstructive pulmonary disease (COPD, GOLD 2B), essential hypertension, peripheral artery disease and ischaemic nephropathy with chronic kidney disease G4 (KDIGO classification). The patient was admitted with COPD exacerbation due to acute tracheobronchitis. However, there was no improvement after almost 3 weeks of antibiotics ( 7 days of amoxicillin/clavulanate and 14 days of piperacillin/tazobactam), optimization of bronchodilators, corticosteroids and respiratory rehabilitation. The patient maintained dyspnoea, cough, sputum retention and wheezing, progressively worsening and needing non-invasive ventilation. Multiple microbiological examinations of sputum were negative. A computed tomography (CT) scan showed an abnormal configuration of the tracheal cartilage: narrowing of the front bow and collapse of the lateral walls inside the lumen (Figs. 1 and 2). 


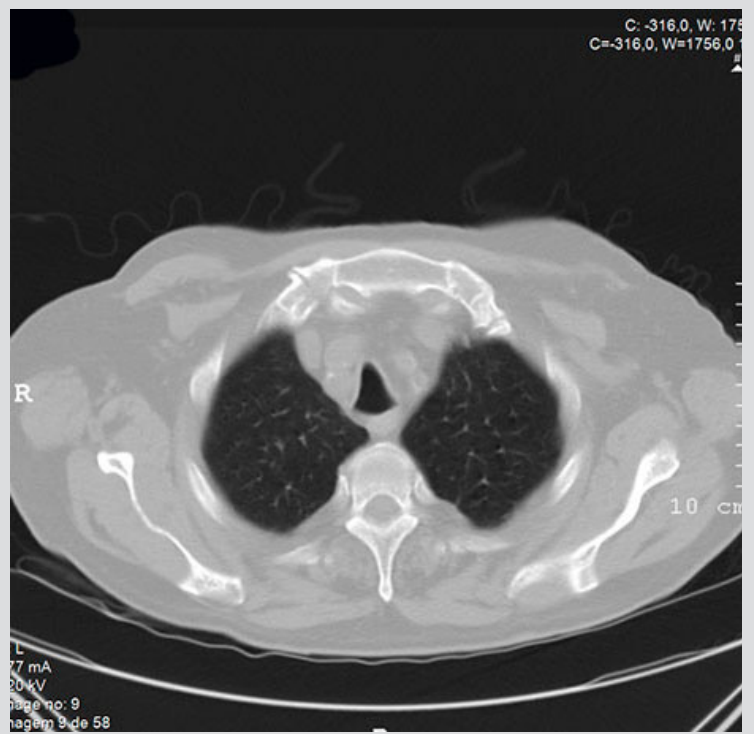

Figure 1. Cross-sectional CT image showing a saber-sheath trachea

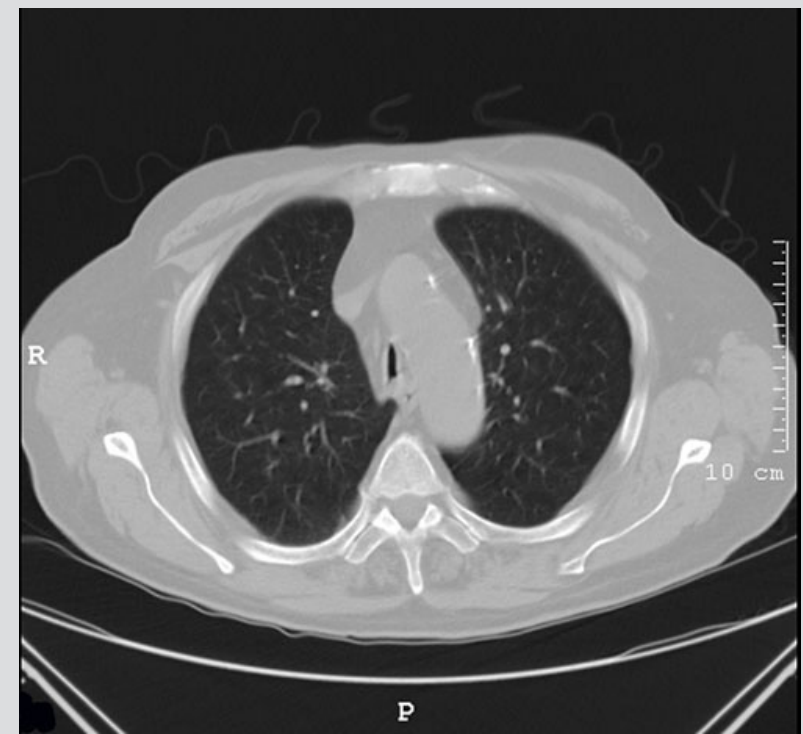

Figure 2. Cross-sectional CT image showing the lateral walls collapsing inside the lumen

Flexible bronchoscopy showed a U-shaped trachea with the upper and lower thirds closed and deviation to the right of the middle third, with partial collapse of the left wall on expiration and coughing, causing a 60-70\% decrease in lumen cross-sectional area, compatible with tracheomalacia, and collapse of right and left main bronchus walls on exhalation, with a decrease in lumen cross-sectional area of 30-40\%. This case was discussed with an interventional bronchology centre which agreed to endotracheal stent placement. At the time, the patient was in an intermediate intensive care unit on non-invasive positive pressure ventilation (NIPPV). Rigid bronchoscopy under jet ventilation showed small tracheal nodules, which were biopsied, and also revealed a saber-sheath trachea in the middle and lower thirds (Fig. 3), without significant obstruction or collapse and, therefore, no indication for tracheal stent placement.

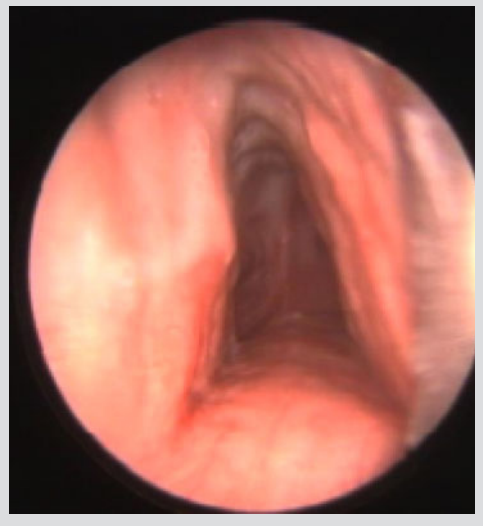

Figure 3. Rigid bronchoscopy image showing a saber-sheath trachea under jet ventilation

The patient was kept on NIPPV. The hospital stay was complicated by nosocomial infections, diverticulitis, kidney failure requiring dialysis, heart failure, delirium and dependency on NIPPV, which led to the patient's death. Later, trachea biopsies showed cartilage degeneration, with no immature characteristics, and mild chronic inflammatory cell infiltration around the cartilage, which is compatible with tracheomalacia. 


\section{DISCUSSION}

Tracheobronchomalacia (TBM) refers to the loss of integrity of airway wall cartilaginous structures resulting in expiratory flow limitation ${ }^{[1,2]}$. It has been identified in 1-4.5\% of all patients undergoing bronchoscopy and has been reported in $23 \%$ of COPD patients. One of the main reasons for the wide range of estimates of the incidence of TBM is the ongoing debate over the definition of the extent of airway collapsibility and morphology required to meet the threshold for pathological collapse. The currently accepted threshold for excessive airway collapse (AC) is a greater than $50 \%$ reduction in airway cross-sectional area with expiration ${ }^{[3]}$.

TBM can be asymptomatic, especially if the airway narrowing is mild. However, as the severity of airway narrowing progresses, the dynamic outflow obstruction leads to symptoms such as dyspnoea, orthopnoea, cough, wheezing, stridor and inability to clear secretions, predisposing the patient to recurrent infections ${ }^{[3,4]}$. The cause of diffuse TBM is often unknown, but it is frequently seen in patients with common respiratory conditions such as asthma and $\mathrm{COPD}^{[3]}$.

The mainstays of diagnosis are dynamic $\mathrm{CT}$ and bronchoscopy with forced expiratory manoeuvres ${ }^{[1,3]}$. Dynamic $\mathrm{CT}$ with end-inspiratory and dynamic-expiratory imaging was shown to reveal a similar degree of AC as bronchoscopy. Given its non-invasiveness, it can be used as the initial test and allows evaluation of the structures around the airways, exclusion of other diseases, and assessment of changes in the lung parenchyma that may cause or be associated with central AC.

Flexible bronchoscopy is the diagnostic gold standard. It allows real-time examination of the airways and accurately captures dynamic airway properties, allows evaluation of the morphology, degree, extent and location of pathology, and allows assessment of response to NIPPV when this is considered an alternative treatment ${ }^{[1]}$. Once TBM is suspected, the patient should undergo both $\mathrm{CT}$ and flexible bronchoscopy to evaluate the degree of $\mathrm{TBM}^{[4]}$.

Initial treatment of symptomatic patients targets the underlying cause of TBM and coexisting conditions ${ }^{[4]}$. In patients who remain symptomatic, NIPPV can be used in the short term to keep the airway open and to facilitate secretion drainage ${ }^{[5]}$. If the patient does not respond to NIPPV, a so-called stent trial can be initiated. If there is improvement, tracheoplasty is offered to operable patients; if patients are not surgical candidates, permanent stent insertion is an alternative, with the understanding that there is a high risk of stent-related adverse effects ${ }^{[1]}$.

This report describes the diagnostic approach and treatment of TBM. Our patient presented with symptomatic TBM, secondary to COPD, even after optimization of medical treatment and NIPPV. Rigid bronchoscopy was performed using jet ventilation, which utilizes a highpressure gas source to keep the airway open and allow the use of a variety of tools. Later, trachea histology confirmed the diagnosis.

Although there is no consensus on the best diagnostic and treatment approach, we believe that in this situation a tracheal stent trial could have been a feasible option. However, we do not know if this procedure would have changed the outcome, since it has stent-related complications like migration, infection, cough and mucus-plugging.

\section{REFERENCES}

1. Murgu S, Colt H. Tracheobronchomalacia and excessive dynamic airwaycollapse. Clin Chest Med 2013;34:527-555.

2. Choo E, Ledford D. Tracheobronchomalacia and hyperdynamic airway collapse. J Allergy Clin Immunol Pract 2015;3:644-645.

3. Buitrago DH, Wilson JL, Parikh M, Majid A, Gangadharan SP. Current concepts in severe adult tracheobronchomalacia: evaluation and treatment. J Thorac Dis 2017;9:E57-E66.

4. Majid A. Tracheomalacia and tracheobronchomalacia in adults. Waltham, MA: UpToDate; 2016. Available from www.uptodate.com (accessed 14 May 2018).

5. Carden KA, Boiselle PM, Waltz DA, Ernst A. Tracheomalacia and tracheobronchomalacia in children and adults - an in-depth review. Chest 2005;127:984-1005. 